\section{Manejo paliativo del cáncer de vesícula biliar irresecable o metastásico: Conclusiones del Consenso Latinoamericano de Manejo del Cáncer de Vesícula Biliar}

\author{
BETTINA MÜLLER ${ }^{1}$, GERARDO ARROYO ${ }^{2}$, JORGE GALLARDO ${ }^{3}$, \\ LUIS VILLANUEVA ${ }^{1}$, PABLO GONZÁLEZ ${ }^{4}$, RAMÓN BAEZA ${ }^{5}$, \\ XABIER DE ARETXABALA ${ }^{6}$, FERNANDO MALUENDA ${ }^{6,9}$, \\ ALEJANDRO ACEVEDO ${ }^{7}$, FERNANDO CHUECAS $^{8}$, JUAN CARLOS DÍAZ $^{9}$, \\ CÉSAR GARCÍA S. ${ }^{10}$, JOSÉ MIGUEL REYES ${ }^{11}$, CLAUDIO NAVARRETE ${ }^{6}$
}

\section{Palliative treatment for locally advanced or metastatic gallbladder cancer: conclusions of the Latin American Consensus meeting for the management of gallbladder cancer}

Gallbladder cancer is a rare disease in Western developed countries, but it is a highly prevalent and lethal disease in Chile and other countries in Latin America. No randomized controlled trials have been performed in gallbladder cancer to establish standard treatments. We therefore performed the first Latin American consensus meeting for the management of gallbladder cancer. In this article we present the conclusions of the panel of experts for the palliative treatment of unresectable or metastatic gallbladder cancer based on a review of the literature, the discussion of the participating experts and the opinion of the assistants. The topics reviewed included: 1.- Gallbladder Cancer and Cholangiocarcinoma-are they the same disease?; 2.- $\mathrm{Pa}$ lliative Chemotherapy: Indications, Drugs and Schedules; 3.- Palliative Radiotherapy; 4.- Palliative Surgery; 5.- Management of Malignant Biliary Obstruction.

(Rev Med Chile 2011; 139: 267-273).

Key words: Antineoplastic protocols; Cholestasis, extrahepatic; Gallbladder neoplasms.

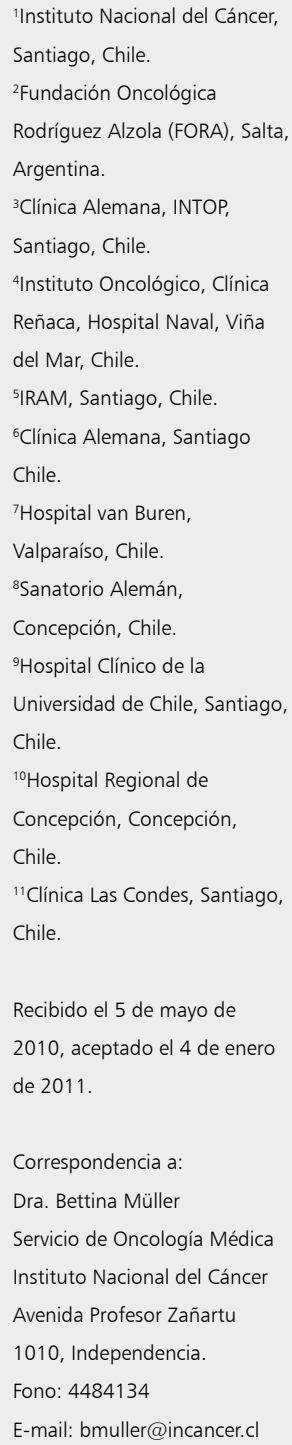

E 1 cáncer de vesícula biliar (CV) es una enfermedad muy poco relevante en Europa y Norteamérica (incidencia en Estados Unidos de Norteamérica (EEUU) de 1,2 por 100.000 habitantes ${ }^{1}$ ), y es huérfano de interés científica, sin embargo, es de alto impacto en áreas de Sudamérica como en Chile, donde constituye la primera causa de muerte por cáncer en mujeres. Particularmente la región de la Araucanía presenta la mayor tasa de mortalidad reportada en el mundo (35 por 100.000 habitantes). Otras regiones con una alta incidencia del cáncer de vesícula biliar son Bolivia, Perú, Ecuador, Colombia, Brasil, la población amerindia de EEUU, Polonia, Algeria e India².

El pronóstico del cáncer de vesícula biliar sigue siendo muy ominoso, con una supervivencia global de sólo $5 \%$ a 5 años ${ }^{3}$. Incluso en pacientes resecados con cirugía curativa en etapas tempranas 
(T2N0M0), la supervivencia a 5 años no llega al $30 \%{ }^{4,5}$, lo cual refleja que un alto porcentaje de los pacientes presentará recurrencias. Muchos pacientes son diagnosticados en etapas avanzadas, fuera del alcance quirúrgico, que constituye la única herramienta curativa.

En el contexto del Simposio Latinoamericano de Gastroenterología Oncológica SLAGO 2009 se desarrolló el primer Consenso Latinoamericano de Cáncer de Vesícula, que abordó los temas de manejo quirúrgico, terapia perioperatoria y terapias paliativas. En el presente texto presentamos las conclusiones referentes a este último tópico, basadas en las presentaciones y votaciones vertidas durante la sesión en el simposio, además de la revisión exhaustiva de la literatura y la discusión del panel de expertos.

\section{¿Los resultados con quimioterapia paliativa reportados en colangiocarcinoma son extrapolables al cáncer de vesícula biliar?}

Tradicionalmente el cáncer de vesícula biliar se ha clasificado dentro de los cánceres de la vía biliar, y como tal se ha incluido en las series de tratamiento de estas patologías. Sin embargo, el CV presenta tasas de respuesta a la quimioterapia (QT) mayores que el colangiocarcinoma (CC) $(34,4 \%$ en $\mathrm{CV}$ versus $20,2 \%$ en CC, $\mathrm{p}=0,008)^{9}$, pero con una supervivencia mediana menor $(7,2$ versus $9,3$ meses, $\mathrm{p}=0,048)^{6-9}$. Por otro lado, existen diferencias en la biología molecular y en el patrón de expresión de blancos moleculares, por ejemplo, las anormalidades de los genes p53 y p16 son más frecuentes en el CC que en el CV ${ }^{40,41}$; mientras que las proteínas Erb2 y EGFR están sobreexpresadas más frecuentemente en $\mathrm{CV}$ que en $\mathrm{CC}^{42}$.

La gran mayoría de los estudios publicados incluye pacientes con $\mathrm{CV}$ y $\mathrm{CC}$, con un número muy reducido por localización ( $<20$ pacientes), lo cual imposibilita un análisis por subgrupo?.

El Consenso recomienda que se realicen estudios clínicos separados o estratificados según localización (colangiocarcinoma intrahepático, extrahepático, vesícula biliar y ampolla de Vater), ya que los resultados no son extrapolables por tratarse de enfermedades distintas.

\section{Indicaciones de la quimioterapia paliativa en cáncer de vesícula biliar}

La QT paliativa tiene la finalidad de mejorar la calidad de vida y aumentar la supervivencia. La supervivencia mediana de pacientes con $\mathrm{CV}$ metastásico sin tratamiento de QT es de 2-4 meses $^{3}$. En los estudios con QT en cáncer de vía biliar se describe una supervivencia mediana de 7-9 meses, utilizando diferentes esquemas de QT incluyendo esquemas basadas en cisplatino (CDDP), basadas en fluropirimidinas, el esquema FAM (5-fluruouracilo (5FU) -doxorubicina- metotrexate) y gemcitabina (Gem) sola o en combinación con fluoropirimidinas o cisplatino ${ }^{6-9}$. Estos resultados son similares a los reportados en una serie chilena de CV avanzado, en la cual se usó QT con $\mathrm{Gem}^{14}$. Glimelius publicó un estudio aleatorizado prospectivo de QT paliativa con los esquemas FLv (5-FU con leucovorina (LV)) o FELv (5-FU, LV y etopósido) versus mejor cuidado de soporte en pacientes con cáncer pancreático y biliar avanzado. El grupo con cáncer biliar mostró un beneficio en supervivencia (6,5 versus 2,5 meses), pero con un total de 37 pacientes incluidos, esta diferencia no fue estadísticamente significativa $(\mathrm{p}=0,1)$. En términos de supervivencia ajustada por calidad de vida se observó un beneficio estadísticamente significativo en el grupo de cáncer biliar $(\mathrm{p}<0,01)^{10}$. En otro estudio aleatorizado controlado se observó una mejoría sintomática en $>50 \%$ de los síntomas estudiados con ambos esquemas de QT usados que consistieron en FELv y ECF (Epirubicina, CDDP y 5FU) ${ }^{11}$.

El Consenso considera indicada la QT paliativa en el cáncer de vesícula biliar metastásico, especialmente en los pacientes con ECOG 0-1, con una reserva funcional adecuada (bilirrubina total inferior a $3 \mathrm{mg} / \mathrm{dl}$, transaminasas menores a 5 veces el valor superior normal, función renal y parámetros hematológicos aceptables) y con lesiones objetivables. No se recomienda en pacientes deteriorados $($ ECOG $>2)$, y en pacientes con falla hepática secundaria a metástasis hepáticas extensas.

\section{Esquemas de quimioterapia}

Se han publicado muy pocos estudios en pacientes con $\mathrm{CV}$ avanzado o metastásico ${ }^{12-17}$. La 
mayoría de los estudios incluyeron a pacientes con CC intra y extrahepático además de CV. Se han estudiado diferentes esquemas de QT; los más ampliamente estudiados fueron fluoropirimidinas \pm platinos o Gem \pm platinos o fluoropirimidinas (Tabla 1).

Se recomienda el esquema de Gem en monodroga, ya que es un esquema bien tolerado, y con tasas de respuesta que fluctúan entre 17,5 y $36 \%$, con una supervivencia mediana reportada entre 7,5 y 13,1 meses en estudios fase II $^{14,18,19}$.
Otra alternativa recomendada es la asociación de Gem con CDDP. Esta combinación presenta una tasa de respuesta entre 17,1 y $64 \%$, con una supervivencia mediana de 8,6 a 10,5 meses ${ }^{9,17,20-22,}$ pero con una toxicidad mayor que la reportada con gemcitabina en monodroga en algunos estudios. Estos dos esquemas fueron comparados en un estudio fase III publicado recientemente, y que constituye el estudio aleatorizado más grande comunicado en cáncer de vía biliar, con 410 pacientes, $36 \%$ de ellos con CV. Se comparó el

\section{Tabla 1}

\begin{tabular}{|c|c|c|c|c|c|}
\hline Autor/año & QT & $\stackrel{n}{\text { (sólo CV) }}$ & $\begin{array}{c}\text { ORR } \\
\text { (CR+PR) }\end{array}$ & os & $\begin{array}{l}\text { Diseño del } \\
\text { estudio }\end{array}$ \\
\hline Yonemoto/2007 [6] & diferentes & 94 de 179 & $10,6 \%$ & $6,5 \mathrm{~m}$ & Serie retrospectiva \\
\hline \multicolumn{6}{|c|}{ Gemcitabina monodroga } \\
\hline Gallardo/2001 [14] & Gem & 26 & $36 \%$ & $7,5 \mathrm{~m}$ & Fase II \\
\hline Park/2005 [18] & Gem & 8 de 23 & $26,1 \% *$ & $13,1 \mathrm{~m}^{*}$ & Fase II \\
\hline Okusaka/2006 [19] & Gem & 22 de 40 & $17,5 \%$ * & $7,6 m^{*}$ & Fase II \\
\hline $\mathrm{Kiba} / 2006[24]$ & Gem & 11 de 39 & $12,8 \% *$ & $17 \mathrm{~m}^{*}$ & Serie retrospectiva \\
\hline \multicolumn{6}{|c|}{ Combinaciones basadas en Gemcitabina } \\
\hline Malik/2003 [17] & Gem/Cis & 11 & $64 \%$ & $10,5 \mathrm{~m}$ & Serie prospectiva \\
\hline Reyes-Vidal/2003 [16] & Gem/Cis & 44 & $48 \%$ & $7 \mathrm{~m}$ & Fase II \\
\hline Doval/2004 [15] & Gem/Cis & 30 & $36,6 \%$ & $5 \mathrm{~m}$ & Fase II \\
\hline Giuliani/2006 [20] & Gem/Cis & 10 de 38 & $32 \%$ * & $8 m^{*}$ & Fase II \\
\hline Meyerhardt/2008 [22] & Gem/Cis & 5 de 33 & $21 \% *$ & $9,7 \mathrm{~m}^{*}$ & Fase II \\
\hline Lee/2008 [21] & Gem/Cis & 14 de 35 & $28,6 \%$ & $8,6 m^{*}$ & Fase II \\
\hline André/2004 [39] & Gem/Ox & 19 de 56 & $54,4 \%$ & $16 \mathrm{~m}$ & Fase II \\
\hline Knox/2005 [7] & Gem/Cap & 22 de 45 & $27 \%$ & $6,6 \mathrm{~m}$ & Fase II \\
\hline \multicolumn{6}{|c|}{ Combinaciones basadas en Fluoropirimidinas } \\
\hline Chatni/2008 [12] & $5 \mathrm{FU} / \mathrm{Cis}$ & 65 & $33,8 \%$ & $5,7 \mathrm{~m}$ & Fase II \\
\hline Nehls/2008 [8] & Cap/Ox & 27 de 65 & $29,6 \%$ & $11,3 \mathrm{~m}$ & Fase II \\
\hline \multicolumn{6}{|c|}{ Comparación esquemas basadas en Gemcitabina vs Fluoropirimidinas } \\
\hline $\mathrm{Kim} / 2008[37]$ & $\begin{array}{l}\text { Gem } \pm \text { Cis ver- } \\
\text { sus FU } \pm \text { Cis }\end{array}$ & 72 de 243 & $\begin{array}{l}16,7 \text { vs } 19,5 \% * \\
\text { (NS) }\end{array}$ & $\begin{array}{c}7,8 \text { vs } 9,1 \mathrm{~m}^{*} \\
\text { (NS) }\end{array}$ & Serie retrospectiva \\
\hline \multicolumn{6}{|c|}{ Estudios randomizados fase III } \\
\hline $\mathrm{Rao} / 2005$ [11] & FELV vs ECF & 26 de 54 & $\begin{array}{c}15 \text { vs } 19,2 \% \text { * } \\
\text { (NS) }\end{array}$ & $\begin{array}{l}12 \text { vs } 9 m^{*} \\
\text { (NS) }\end{array}$ & $\begin{array}{l}\text { Fase III, se cerró por } \\
\text { falta de enrolamiento }\end{array}$ \\
\hline Valle/2010 [23] & $\begin{array}{l}\text { Gem versus } \\
\text { Gem/Cis }\end{array}$ & 149 de 410 & $\begin{array}{l}15,9 \text { vs } 25,7 \% * \\
\text { (NS) }\end{array}$ & $\begin{array}{c}8,1 \text { vs } 11,7 m^{*} \\
(p=0,002)\end{array}$ & Fase III \\
\hline
\end{tabular}

*resultados para el grupo total, no separado por VB vs CC. (ORR: respuesta global, CR: respuesta completa, PR: respuesta parcial, OS: supervivencia global, m: meses) 
esquema de Gem $1.000 \mathrm{mg} / \mathrm{m} 2 \mathrm{D} 1+8+15$ cada 21 días por un máximo de 6 ciclos ( $=24$ semanas) con el esquema de CDDP $25 \mathrm{mg} / \mathrm{m}^{2}$ seguido por Gem $1.000 \mathrm{mg} / \mathrm{m}^{2} \mathrm{D} 1+8$ cada 21 días por un máximo de 8 ciclos (= 24 semanas). La combinación de Gem + CDDP (GemCis) fue superior en supervivencia global ( 11,7 vs 8,1 meses, p < 0,001, HR 0,64, 95\% CI: $0,52-0,80)$, y en supervivencia libre de progresión ( 8,0 vs 5,0 meses, $\mathrm{p}<0,001$, HR 0,63, $95 \% \mathrm{CI}$ : $0,51-0,77)$. A diferencia de lo sugerido por estudios fase II, la toxicidad de ambos esquemas fue similar, con $57 \%$ de toxicidad grado 3 ó 4 en cada rama ${ }^{23}$. Este estudio establece el esquema GemCis como nuevo estándar en el tratamiento del cáncer de vía biliar. En el análisis por subgrupo de $\mathrm{CV}$, se mantuvo dicho beneficio.

Para definir el esquema a usar se deben tomar en cuenta las características del paciente, el perfil de toxicidad del fármaco y aspectos socioeconómicos.

La terapia se podría mantener hasta la progresión de la enfermedad o la aparición de toxicidad inaceptable, aunque la duración óptima de la QT paliativa aún no ha sido establecida.

La QT de segunda línea estaría indicada sólo en casos muy seleccionados, idealmente en el contexto de estudios clínicos. Un análisis retrospectivo reportó tasas de respuesta y una supervivencia mediana similares al usar Gem en primera versus segunda línea, lo cual podría indicar que existe un subgrupo de pacientes con una evolución clínica más favorable, en los cuales se podría plantear una QT de segunda línea ${ }^{24}$. Sin embargo, esta observación no pudo ser reproducida en una serie prospectiva de 33 pacientes con cáncer de la vía biliar que recibieron QT de segunda línea, mayoritariamente con Gem, sin que se observara una respuesta objetiva ${ }^{8}$.

El Consenso recomienda los siguientes esquemas de QT de primera línea:

1. Gemcitabina + Cisplatino

2. Gemcitabina monodroga

\section{Rol de la radioterapia paliativa}

La radioterapia (RT) paliativa se podría plantear para el manejo sintomático de la enfermedad localmente avanzada. Existen escasos datos acerca del beneficio de la RT paliativa, pero podría ayudar a aliviar síntomas como la ictericia, el dolor y el prurito $^{25}$. La RT en CV es bien tolerada según las series publicadas ${ }^{26,27}$. Flickinger reportó una serie de 8 pacientes irresecables o recurrentes, tratados con RT paliativa, cuya supervivencia mediana fue de 3 meses ${ }^{27}$. La tasa de respuesta objetiva en una serie de 17 pacientes irresecables fue de $24 \%{ }^{28}$.

El Consenso recomienda considerar la RT paliativa en casos seleccionados y basado en la decisión de un Comité Oncológico Multidisciplinario.

\section{Rol de la cirugía en el cáncer de vesícula biliar avanzado}

Dada la pobre supervivencia en este grupo de pacientes, la cirugía se debe emplear en forma cautelosa. En presencia de metástasis peritoneales o hepáticas no tendría indicación ${ }^{29}$. La derivación bilio-digestiva puede aliviar la ictericia en caso de obstrucción de la vía biliar. En una serie de 41 pacientes con ictericia, prurito y colangitis tratados con derivación bilio-digestiva, se reportaron altas tasas de mortalidad (12\%) y morbilidad (51\%) perioperatoria, por lo que se debería reservar este procedimiento para casos seleccionados ${ }^{31}$. En presencia de un síndrome de mal vaciamiento gástrico secundario, puede plantearse una gastroyeyunostomía paliativa ${ }^{30}$. Las resecciones extensas, incluyendo la hepatectomía con o sin resección de la vía biliar, y linfadenectomía sólo están indicadas si se puede lograr una resección R0. Su utilidad en tumores localmente avanzados es dudosa, ya que no mejoraría la supervivencia en etapa $\mathrm{T} 4^{32}$. Si bien se han reportado casos promisorios ${ }^{38}$, no existe suficiente evidencia que el rescate quirúrgico luego de la QT paliativa con respuesta parcial aumente la supervivencia. En casos seleccionados se puede plantear, siempre y cuando se pueda lograr una resección R0 con morbimortalidad razonable. Este tipo de intervenciones sólo se deben realizar por equipos quirúrgicos experimentados.

El Consenso recomienda considerar la cirugía en casos seleccionados, basado en la evaluación por un Comité Oncológico Multidisciplinario. 


\section{Rol de la colangiografía retrograda endoscópica y uso stent}

La obstrucción maligna de la vía biliar puede causar prurito, malabsorción, colangitis y falla hepática, por lo que está indicado realizar procedimientos de descompresión paliativa. Previamente debe descartarse la presencia de metástasis hepáticas múltiples con falla hepática secundaria como causa del cuadro ictérico. Por tratarse de procedimientos invasivos, deben ser indicados con cautela en pacientes deteriorados $(\mathrm{ECOG}>2)$.

Existen dos métodos de descompresión de la vía biliar: la cirugía y la colocación de un stent. Este último procedimiento se asocia a una menor morbi-mortalidad, por lo que se considera la terapia estándar actualmente ${ }^{33}$. Cuando la vía endoscópica no es factible, se puede plantear la inserción de un stent vía percutánea ${ }^{34}$.

En los pacientes con una expectativa de vida mayor a 4 meses, podría ser preferible el uso de stents metálicos autoexpansibles por sobre los de polietileno, por una mayor tasa de éxito en descomprimir la vía biliar y una menor tasa de re-oclusión con necesidad de recambio ${ }^{35}$. Los stents metálicos cubiertos presentan menor incidencia de oclusión, con menos reintervenciones, pero sin impacto en la supervivencia de los pacientes tratados ${ }^{36}$.

El consenso recomienda los siguientes procedimientos para el manejo de la ictericia obstructiva maligna:

1. Stent endobiliar insertado bajo visión ERCP

2. Stent endobiliar insertado vía transparietohepática

\section{Referencias}

1. Carriaga MT, Henson DE. Liver, gallbladder, extrahepatic bile ducts, and pancreas. Cancer 1995; 75 (1 Suppl): $171-90$

2. Lazcano-Ponce E, Miquel JF, Muñoz N, Herrero R, Ferrecio $\mathrm{C}$, Wistuba I, et al. Epidemiology and Molecular Pathology of Gallbladder Cancer. CA Cancer J Clin 2001; 51: 349-64.

3. Cubertafond P, Gianant A, Cucchiaro G. Surgical treatment of 724 carcinomas of the gallbladder: Results of the French Surgical Association Survey. Ann Surg 1994; 219 (3): 275-80.

4. Greene FL, Page DL, Fleming ID, Fritz A, Balch CM, Haller DG, Morrow M. AJCC Cancer Staging Manual, 6th ed. New York: Springer, 2002.

5. Fong Y, Wagman L, Gonen M, Crawford J, Reed W, Swanson R, et al. Evidence-Based Gallbladder Cancer Staging - Changing Cancer Staging by Analysis of Data From the National Cancer Database. Ann Surg 2006; 243 (6): 767-74.

6. Yonemoto N, Furuse J, Okusaka T, Yamao K, Funakoshi A, Ohkawa S, et al: A Multi-center Retrospective Analysis of Survival Benefits of Chemotherapy for Unresectable Biliary Tract Cancer. Jpn J Clin Oncol 2007; 37 (11): 843-51.

7. Knox JJ, Hedley D, Oza A, Feld R, Siu LL, Chen E, et al. Combining Gemcitabine and Capecitabine in Patients With Advanced Biliary Cancer: A Phase II trial. J Clin Oncol 2005; 23 (10): 2332-8.

8. Nehls O, Oettle H, Hartmann JT, Hofheinz RD, Hass HG, Horger MS, et al. Capecitabine plus oxaliplatin as first-line treatment in patients with advanced biliary system adenocarcinomaa: a prospective multicentre phase II trial. Br J Cancer 2008; 98 (2): 309-15.

9. Eckel F, Schmid RM: Chemotherapy in advanced biliary tract carcinoma: a pooled analysis of clinical trials. $\mathrm{Br} \mathrm{J}$ Cancer 2007; 96: 896-902.

10. Glimelius B, Hoffman K, Sjödén PO, Jacobsson G, Sellström $\mathrm{H}$, Enander LK, et al: Chemotherapy improves survival and quality of life in advanced pancreatic and biliary cancer. Ann Oncol 1996; 7 (6): 593-600.

11. Rao S, Cunningham D, Hawkins RE, Hill ME, Smith D, Daniel F, et al. Phase III study of 5FU, etoposide and leucovorin (FELV) compared to epirubicin, cisplatin and 5FU (ECF) in previously untreated patients with advanced biliary cancer. Br J Cancer 2005; 92: 1650-4.

12. Chatni SS, Sainani RS, Mehta SA, Mohandes KM. Infusion chemotherapy with cisplatinum and fluorouracil in the treatment of locally-advanced and metastatic gallbladder cancer. J Cancer Res Ther 2008, 4 (4): 151-5.

13. Julka PK, Puri T, Rath GK. A phase II study of gemcitabine and carboplatin combination chemotherapy in gallbladder carcinoma. Hepatobiliary Pancreat Dis Int 2006; 5 (1): 110-4.

14. Gallardo J, Rubio B, Fodor M, Orlandi L, Yañez M, Gamargo C, Ahumada M: A phase II study of gemcitabine in gallbladder carcinoma. Ann Oncol 2001; 12: 1403-6.

15. Doval DC, Sekhon JS, Gupta SK, Fuloria J, Shukla VK, Gupta $S$ et al. A Phase II study of gemcitabine and cisplatin in chemotherapy-naïve, unresectable gallbladder cancer. Br J Cancer 2004; 90: 1516-20. 
16. Reyes-Vidal JM, Gallardo J, Yáñez E, Rosas J, Palma M, Cerda B, et al. Gemcitabine (Gem) and Cisplatin (CIS) in the treatment of patients (pts) with unresectable or metastatic gallbladder cancer: Results of the phase II GOCCHI study 2000-13. Proc Am Soc Clin Oncol (2003) 22:273 abstr 1095.

17. Malik I, Aziz Z, Zaidi SH, Manzoor MD, Sethuraman G. Gemcitabine and Cisplatin Is a Highly Effective Combination Chemotherapy in Patients With Advanced Cancer of the Gallbladder, Am J Clin Oncol 2003; 26 (2): 174-7.

18. Park JS, Oh SY, Kim SH, Kwon HC, Kim JS, Jin-Kim H, Kim YH: Single-agent Gemcitabine in the Treatment of Advanced Biliary Tract Cancers: a Phase II Study. Jpn J Clin Oncol 2005; 35 (2): 68-73.

19. Okusaka T, Ishii H, Funakoshi A, Yamao K, Ohkawa S, Saito S, et al: Phase II study of single-agent gemcitabine in patients with advanced biliary tract cancer. Cancer Chemother Pharmacol 2006; 57 (5): 647-53.

20. Giuliani F, Gebbia V, Maiello E, Borsellino N, Bajardi E, Colucci G: Gemcitabine and cisplatin for inoperable and/or metastatic biliary tree carcinomas: a multicenter phase II study of the Gruppo Oncologico dell'Italia Meridionale (GOIM). Ann Oncol 2006; 17 Suppl 7: vii73-77.

21. Lee J, Kim TY, Lee MA, Ahn MJ, Kim HK, Lim HY, et al: Phase II trial of gemcitabine combined with cisplatin in patients with inoperable biliary tract carcinoma. Cancer Chemother Pharmacol 2008; 61 (1): 47-52.

22. Meyerhardt JA, Zhu AX, Stuart K, Ryan DP, Blaskowsky L, Lehman N, et al: Phase-II Study of Gemcitabine and Cisplatin in Patients with Metastatic Biliary and Gallbladder Cancer. Dig Dis Sci 2008; 53 (2): 564-70.

23. Valle J, Wasan H, Palmer DH, Cunningham D, Anthoney A, Maraveyas A, et al Cisplatin plus Gemcitabine versus Gemcitabine for Biliary Tract Cancer. N Engl J Med 2010; 362; 14: 1273-81.

24. Kiba T, Nishimura T, Matsumoto S, Hatano E, Mori A, Ysaumi S, et al: Single -Agent Gemcitabine for Biliary Tract Cancer. Oncology 2006; 70 (5): 358-65.

25. Houry S, Barrier A, Huguier M: Irradiation therapy for gallbladder carcinoma: recent advances. J HepatoBiliary-Pancreat Surg 2001; 8 (6): 518-24.

26. Hanna SS, Rider WD: Carcinoma of the gallbladder or extrahepatic bile ducts: the role of radiotherapy. Can Med Assoc J 1978; 118 (1): 59-61.

27. Flickinger JC, Epstein AH, Iwatsuki S, Carr BI, Starzi TE. Radiation therapy for primary carcinoma of the extrahepatic biliary system. An analysis of 63 cases. Cancer 1991; 68 (2): 289-94.

28. Uno T, Itami J, Aruga M, Araki H, Tani M, Kobori O.
Primary carcinoma of the gallbladder: role of external beam radiation therapy in patients with locally advanced tumor. Strahlenther Onkol 1996; 172 (9): 496-500.

29. Maluenda F, Díaz JC, Aretxabala X, Burdiles P, Csendes A, Contreras L. Aspectos quirúrgicos del cáncer de vesícula biliar. Rev Med Chile 2005; 133: 723-8.

30. Gourgiotis S, Kocher HM, Solaini L, Yarollahi A, Tsiambas E, Salemis N. Gallbladder cancer. Am J Surg 2008; 196 (2): 252-64.

31. Kapoor VK, Pradeep R, Haribhakti SP, Singh V, Sikora SS, Saxena R, Kaushik SP: Intrahepatic segment III cholangiojejunostomy in advanced carcinoma of the gallbladder. Br J Surgery 1996; 83 (12): 1709-11.

32. Shih SP, Schulick RD, Cameron JL, Lillemoe KD, Pitt HA, Choti MA, et al. Gallbladder Cancer: The Role of Laparoscopy and Radical Resection. Ann Surg 2007; 245 (6): 893-901.

33. Smith AC, Dowsett JF, Russell RC, Hatfield AR, Cotton $\mathrm{PB}$, Randomised trial of endoscopic stenting versus surgical bypass in malignant low bileduct obstruction. Lancet 1994; 344 (8938): 1655-60.

34. Piñol V, Castells A, Bordas JM, Real MI, Llach J, Montañà $\mathrm{X}$, et al. Percutaneous Self-expanding Metal Stents versus Endoscopic Polyethylene Endoprostheses for Treating Malignant Biliary Obstruction: Randomized Clinical Trial. Radiology 2002; 225 (1): 27-34.

35. Guo YX, Li YH, Chen Y, Chen PY, Luo PF, Li Y, et al. Percutaneous transhepatic metal versus plastic biliary stent in treating malignant biliary obstruction: a multiple center investigation. Hepatobiliary Pancreat Dis Int 2003; 2 (4): 594-7.

36. Isayama $\mathrm{H}$, Komatsu $\mathrm{Y}$, Tsujino $\mathrm{T}$, Sasahira $\mathrm{N}$, Hirano $\mathrm{K}$, Toda $\mathrm{N}$, et al. A prospective randomised study of "covered" versus "uncovered" diamond stents for the management of distal malignant biliary obstruction. Gut 2004; 53: 729-34.

37. Kim ML, Oh DY, Lee SH, Kim DW, Im SA, Kim TY, et al. Gemcitabine-based versus fluoropyrimidine-based chemotherapy with or without platinum in unresectable biliary tract cancer: a retrospective study. BMC 2008, 8: 374.

38. Gallardo JO, Rubio B, Díaz JC, Maluenda F. Combined chemotherapy and surgery for metastatic gallbladder cancer improve median survival. J Clin Oncol 2006; 24 (18S) 14136.

39. André T, Tournigand C, Rosmorduc O, Provent S, Maindrault-Goebel F, Avenin D, et al. Gemcitabine combined with oxaliplatin (GEMOX) in advanced biliary tract adenocarcinoma: a GERCOR study. Ann Oncol 2004, 15 (9): 1339-43.

40. Della Torre G, Pasquini G, Pilotti S, Alasio L, Civelli E, 
Cáncer de vesícula biliar: Consenso Latinoamericano - B. Müller et al

Cozzi G, et al. TP53 mutations and $\mathrm{mdm} 2$ protein overexpression in cholangiocarcinomas. Diagn Mol Pathol 2000; 9 (1): 41-6.

41. Wistuba II, Albores-Saavedra J. Genetic abnormalities involved in the pathogenesis of gallbladder carcinoma. J
Hepatobiliary Pancreat Surg 1999; 6 (3): 237-44.

42. Nakazawa K, Dobashi Y, Suzuki S, Fujii H, Takeda Y, Ooi A: Amplification and overexpression of c-erbB-2, epidermal growth factor receptor, and c-met in biliary tract cancers. J Pathol 2005; 206 (3): 356-65. 